\title{
Investigational Subject Case History
}

National Cancer Institute

\section{Source}

National Cancer Institute. Investigational Subject Case History. NCI Thesaurus. Code C142588.

The detailed documentation about an individual in a clinical trial that is compiled and managed by the investigator. This includes information on informed consent, medical records and other observations. 